\title{
Introduction: Endovascular approaches to cerebral ischemia
}

\author{
Aaron S. Dumont, M.D., and Avery J. Evans, M.D. \\ Departments of Neurological Surgery and Radiology, University of Virginia Health System, \\ Charlottesville, Virginia
}

\begin{abstract}
7 He treatment of stroke has rapidly evolved over the past decade, particularly as data concerning the natural history have emerged and endovascular treatment options have matured. Therapies for acute stroke have expanded as intraarterial thrombolysis and mechanical clot retrieval have been validated. Furthermore, angioplasty and stenting of intra- and extracranial vessels have evolved with the development of new devices coupled with increasing operator experience. Endovascular therapy is now a widely accepted treatment option for cerebral ischemia in many clinical situations.
\end{abstract}

The March 2009 issue of Neurosurgical Focus is devoted to the endovascular management of cerebral ischemia. The first article from Dr. Prestigiacomo's group provides a comprehensive review of the endovascular management of acute ischemic stroke. They provide an excellent review of the literature, defining the role of intraarterial thrombolysis, mechanical thrombectomy, and intracranial angioplasty and stenting in the management of acute ischemic stroke. The article by Kole and colleagues presents a series of patients with nonacute intracranial occlusions and near occlusions treated with intracranial angioplasty and stent placement. They offer a timely contribution that illustrates the feasibility and potential effectiveness of this strategy in selected patients. This article is also accompanied by a podcast for further detail and emphasis. The next article provides a contemporary discussion of a feared complication of endovascular revascularization, namely hyperperfusion syndrome.
The experienced cerebrovascular group at Northwestern University, in the next article, provides a discussion of the endovascular management of delayed cerebral ischemia following SAH. Eddleman and colleagues specifically provide a focused review of the literature and specify their current management protocol. The final article presents an interesting case of a traumatic vertebral-venous fistula following a gunshot wound in which the patient presented with delayed cerebral ischemia; the authors outline the endovascular management unique to this case.

$$
\text { sox }
$$

Endovascular therapy is a rapidly expanding and continuously evolving area in the management of patients with stroke. It is hoped that the present issue of Neurosurgical Focus highlights many of the recent developments in this area for the general readership and also emphasizes the future importance of endovascular therapy for the treatment of stroke. 